\title{
Platelet-Rich Plasma Therapy for Knee Joint Problems: Review of the Literature, Current Practice and Legal Perspectives in Korea
}

\author{
Yong-Geun Park, $\mathrm{MD}^{1}$, Seung Beom Han, $\mathrm{MD}^{2}$, Sang Jun Song, $\mathrm{MD}^{3}$, Tae Jin Kim, $\mathrm{MD}^{3}$ and Chul-Won Ha, \\ $\mathrm{MD}^{1}$ \\ Department of Orthopaedic Surgery, ${ }^{1}$ Samsung Medical Center, Sungkyunkwan University School of Medicine; ${ }^{2}$ Anam Hospital, Korea University College of Medicine; \\ ${ }^{3}$ Kyung Hee University School of Medicine, Seoul, Korea
}

Platelet-rich plasma (PRP) is a concentrate extract of platelets from autologous blood, and represents a possible treatment option for the stimulation and acceleration of soft-tissue healing and regeneration in orthopedics. Currently, the availability of devices for outpatient preparation and delivery contributes to the increase in the clinical use of PRP therapy in practical setting of orthopedic fields. However, there is still paucity of scientific evidence in the literature to prove efficacy of PRP therapy for the treatment of ligament or tendon problems around the knee joint. Moreover, strong evidence from well-designed clinical trials to support the PRP therapy for osteoarthritis of the knee joint is yet scanty in the literature. Scientific studies need to be performed to assess clinical indications, efficacy, and safety of PRP, and this will require high powered randomized controlled trials. Nonetheless, some hospitals exaggeratedly advertise PRP procedures as the ultimate treatment and a novel technology with abundant scientific evidence for the treatment of knee problems. As a matter of fact, PRP protocols are currently approved only for use in clinical trials and research, and are not allowed for treatment purpose by any institutions in Korea. At present, clinical use of PRP therapy for ligament or tendon problems or osteoarthritis of knee joint is defined as illegal medical practice, regardless of whether it is performed as a sole procedure or as a part of prolotherapy, because the safety and validity are not yet approved by the Ministry of Health and Welfare and Health Insurance Review and Assessment Service. Practicing physicians should remember that injection of PRP to patients by imposing medical charge is still illegal as per the current medical law in Korea.

Key words: Platelet-rich plasma, Osteoarthritis, Ligament, Tendon, Knee joint.

\section{Introduction}

Platelet-rich plasma (PRP) is a concentrate extract of platelets from autologous blood. It is known to increase growth factor concentration three to five times of normal plasma and helps

Received April 17, 2012; Accepted April 26, 2012.

Correspondence to: Chul-Won Ha, MD.

Department of Orthopaedic Surgery, Samsung Medical Center, Sungkyunkwan University School of Medicine, 81 Irwon-ro, Gangnamgu, Seoul 135-710, Korea.

Tel: +82-2-3410-0275, Fax: +82-2-3410-0061

Email: hacw@skku.edu

This is an Open Access article distributed under the terms of the Creative Commons Attribution Non-Commercial License (http://creativecommons.org/licenses/by-nc/3.0/) which permits unrestricted non-commercial use, distribution, and reproduction in any medium, provided the original work is properly cited. to heal the injured tissue $e^{1,2)}$. Its use is reported in the fields of dermatology, plastic surgery, dentistry, otolaryngology, urology, ophthalmology, and neurosurgery. In the fields of orthopedics and sports medicine, some clinicians have applied PRP therapy in acute ligament injury (medial collateral ligament rupture of knee joint, lateral collateral ligament rupture of ankle, etc.), chronic tendon problems (rotator cuff tear, lateral epicondylitis of elbow, tendinitis around the knee joint, Achilles tendinitis, plantar fasciitis, etc.), acute muscle rupture, or facilitation of bone healing after bone graft. Experimentally, it has also been applied in intraarticular injections for chondromalacia or osteoarthritis ${ }^{1-5)}$. However, most clinical reports on PRP in orthopedics and sports medicine are case reports or case-series studies without a control group (evidence level IV), or do not have enough statistical significance due to their small sample size ${ }^{2)}$. Since most growth factors are secreted within one hour after intraarticular PRP injection, PRP cannot alter the pathophysiology of chronic diseases such as osteoarthritis, and it is difficult to expect that 
cartilage that is already worn out will regenerate with that short exposure to growth factors.

Related institutions such as the Ministry of Health and the National Evidence-based Healthcare Collaborating Agency have stated their position that it is illegal to perform PRP procedures and charge patients for the procedures whose safety and efficacy have not yet been proven. Nevertheless, PRP procedures are receiving the spotlight as a new profit-making model, and several hospitals are advertising about such procedures through the internet and other media.

It is a big concern in terms of socio-economic costs and national health levels that PRP procedures are spreading indiscreetly without sufficient medical evidence. Moreover, even the safety of the procedures has not yet been fully established. Furthermore, PRP procedures are being abused by some hospitals as a means of unjust profit-making and hospital advertisement, which can transfer relative disadvantages to the majority of medical institutions that follow honest evidence-based medicine. It is important to note that although some novel medical technologies develop and improve through critics and mistakes, many are discarded due to problems in safety and validity ${ }^{6,7)}$.

Therefore, we enclosed the current situation of PRP-related exaggerative advertisements in Korea, review of the current literatures to discover what scientific evidence we currently do have regarding the PRP therapy around the knee joint, and the perspectives of the Korean regulating organizations in terms of the legal issues in this review. Accordingly, we would like to provide a reference for the practicing physicians with regard to the clinical applications and researches with PRP.

\section{The Current Situation of PRP-related Exaggerative Advertisements in Korea}

Advertisements of PRP procedures can be found in newspapers, TV broadcasts, and hospital promotion sites. They are mostly found on digital free newspapers, internet versions of major newspapers, and hospital promotion sites. In addition, information in certain advertisements are being quoted in blogs, knowledge sites, and Q\&A sites, making it difficult for nonmedical public to judge such contents objectively. The titles and contents of these advertisements describe PRP as the primary or ultimate treatment for the certain disease mentioned in the advertisement, and PRP is described as a cutting-edge medical technology with scientific evidence.

The problems regarding those advertisement contents can be summarized as the followings: First, the number of PRP-related articles is increasing. Starting from the article introducing PRP prolotherapy in March, 2009 (OSEN entertainments 2009-3-21), the number is increasing annually: 10 articles in 2009, 168 articles in 2010, and 248 articles in 2011.

Second, the advertised indications for PRP procedures are broadening, and the therapeutic benefits being described are becoming more and more exaggerated. One article in April 2009 stated that PRP prolotherapy may be an alternative treatment in acute injury of the hamstring (tendon) (OSEN living/culture 2009-4-23). However, in 2011, there were articles that stated PRP as the (a) fundamental therapy for cartilage strengthening (Segye Press 2011-04-11), that meniscus tear is treatable by PRP without surgery (Sports world 2011-08-28), that 3 injections of PRP in cartilage injury can eliminate pain (Health Chosun 2011-10-04), and that avascular necrosis of the femoral head can also be completely healed with PRP (Sports world 2011-12-14). These examples are only to mention a few, and other numerous indications and exaggerated therapeutic benefits are being reported with hospital advertisements.

Third, PRP-related advertisements are concentrated on only a few hospitals. Over $90 \%$ of PRP-related articles in the orthopedic and sports medicine area are from ' $\mathrm{Y}$ ' hospital, ' $\mathrm{T}$ ' hospital, 'L' clinic, 'S' clinic, etc. It is worrisome that the majority of medical institutions, which do not perform PRP procedures due to the lack of validity and legal restrictions, may be perceived by the public as "not up to date".

Fourth, the so-called evidence most PRP-related articles bring up as the basis of their contents was game results of overseas sports stars who have received PRP procedures (such as Tiger Woods, Hines Ward, Kobe Bryant) or case reports published on small foreign journals reported by doctors who performed such PRP procedures ${ }^{8}$. It is difficult for the general public to discern whether the effects of PRP procedures described in these articles are really based on scientific objectivity.

Fifth, articles about results of PRP-related research done by a few academic institutions are quoted in other articles. The research results of PRP application to knee cartilage diseases by the Catholic University Uijeongbu St. Mary's Hospital (Medicaltoday 2011-08-12) were quoted 4 times in other articles, and the research results of PRP application to rotator cuff tear by Kwandong University Myongji Hospital (Segyeilbo 2011-05-16) were quoted 3 times in other articles. We must remind ourselves that reports of PRP-related research results from academic institutions described in these articles could be accepted by the public, who do not have an idea on clinical research and have limited indications and circumstances, as evidence that proves 
the safety and validity of PRP procedures for most orthopedic problems.

What is fortunate is that there are some articles that point out the validity and legal aspects of PRP procedures. Examples include the article quoting New York Times and explains that PRP procedures are not effective (Chosunilbo 2011-09-09), articles that reported the abusive use of PRP procedures (Money Today 2011-09-29, Medipharm News 2011-10-19, Korean Healthlog 2011-10-23), articles saying that PRP procedures have no medical evidence (Doctor's News, Money Today, Korea Health Log 2011-11-03), and the article saying that PRP procedures are illegal under current medical law (Korea Health Log 2011-0930, 2011-10-19). These articles could be used by hospitals that do not perform PRP procedures when patients demand such procedures.

Advertising uncertain material about PRP procedures through the press and wrongly applying such procedures to general patients is certainly a harmful deed both in the socioeconomic point of view and the national public health point of view. Such deeds also make the public see honest hospitals that do not perform such procedures as if they are incompetent hospitals that do not care about novel medical technologies. PRP is yet a new procedure only in the early research stage. Its effectiveness is not medically proven, and especially so in chronic diseases such as degenerative osteoarthritis. Arguments about the efficacy of PRP in degenerative osteoarthritis should be made carefully and only in the academic research field. Carelessly advertising such material to the public can be an act of false or exaggerated advertisement without medical evidence.

Therefore, efforts must be made by medical facilities, the press, and related government institutions to clear away such false or exaggerated material. Also, it would be reasonable that only papers describing clinical results collected with patient consent under strict control of institutional review boards and published by academic journals make it into general newspaper articles.

\section{PRP: Literature Review of Clinical Use around Knee Joint}

PRP represents a possible treatment option for the stimulation and acceleration of soft-tissue healing and regeneration. Use of PRP treatment has expanded in the field of sports medicine because it is autogenously originated and easily prepared. According to the recently reported literature reviews, the level of current research lacks enough scientific evidence to prove safety and effectiveness of PRP treatment, and it emphasized that more research needs to be carried out with randomized, doubleblind methods ${ }^{2,9-13)}$. Despite the lack of scientific evidence on the effectiveness of PRP, the availability of devices for outpatient preparation and delivery of PRP has contributed to the increase in the clinical use of PRP treatment.

\section{PRP for Ligaments or Tendons around Knee Joint}

The healing process of ligament and tendon is extremely complex and not fully understood with regard to the actions among stimulatory, inhibitory, and regulatory factors ${ }^{14)}$. In this setting, it has not been fully recognized how and when injection of growth factor or PRP into damaged tissue stimulates the healing process. Kajikawa et al. ${ }^{14)}$ reported that PRP increased the number of collagen fiber and fibroblast in the early phase of healing process after the damage to tendon of rats. PRP is known to stimulate the synthesis of hepatocyte growth factor (HGF) and vascular endothelial growth factor (VEGF) present in the tendon cells, which in turn increases cellular proliferation and vascular regeneration. However, the balance between transforming growth factor- $\beta 1$ (TGF- $\beta 1$ ) and the pools of platelet-secreted molecules may have important therapeutic implications in the control of angiogenesis and fibrosis ${ }^{15)}$. Murray et al. ${ }^{16)}$ reported that addition of PRP to the suture repairs of anterior cruciate ligament (ACL) did not improve anterior-posterior knee laxity, maximum tensile load, or linear stiffness of the ACL repairs after 14 weeks in vivo. They concluded that use of PRP alone to supplement suture repair of the ACL was ineffective in the animal model and they were confident that a clinically meaningful effect as a result of adding PRP was unlikely.

Although there have not been enough clinical research on the effects of growth factor and PRP on chronic tendon injury, it has been considered to be used for chronic tendinopathy due to the need for effective conservative therapies and basic research result of its effectiveness on angiogenesis and collagen synthesis ${ }^{3,17}$. Filardo et al. ${ }^{18)}$ compared PRP injection group $(n=15)$ to the control group $(n=16)$, for patients with chronic jumper's knee who had failed previous nonsurgical or surgical treatments regarding, Tegner scale, EQ-Visual Analogue Scale (EQ-VAS) scale, and pain level. Even though the group with PRP injection showed higher scores on all the criteria, its outcome indicated no statistical significance when compared to the control group. Coombes et al. ${ }^{19)}$ performed a systematic review of randomized trials to establish clinical efficacy and risk of adverse events for treatment by injection. The result of the analysis of 2672 participants showed that PRP were not more efficacious than was placebo for Achilles tendinopathy, while 
prolotherapy was not more effective than was eccentric exercise. $\mathrm{Hart}^{20)}$ also performed a database search using terms describing common tendinopathies and injections, including steroids, local anesthetics, sclerosing solutions, aprotinin, PRP, botulinum toxins, and glycosaminoglycans to determine the efficacy and risk of adverse effects of peritendinous corticosteroid and other injections in the management of tendinopathy. Based on the review of 174 papers, they concluded that corticosteroid injection is beneficial in the short term for the treatment of tendinopathies but may be worse than other treatments in the intermediate and long terms, and no clear evidence of benefit of other injections was shown, except for sodium hyaluronate in the short and long term in overall improvement and pain reduction of lateral epicondylalgia (1 study). Therefore, the effect of PRP injections for chronic tendinopathy is currently not proven. de Vos et al. ${ }^{21)}$ revealed in their report of systemic review of autologous growth factor injection (whole blood or PRP) for chronic tendinopathy that although all studies showed that injections of autologous growth factors (whole blood and PRP) in patients with chronic tendinopathy had a significant impact on improving pain and/ or function over time, only three studies using autologous whole blood had a high methodological quality assessment, and none of them showed any benefit of an autologous growth factor injection when compared with a control group. They emphasized that there was strong evidence that the use of injections with autologous whole blood should not be recommended. They also emphasized that there were no high quality studies found on PRP treatment, and thus, there was limited evidence to support the use of injections with PRP in the management of chronic tendinopathy. Currently, there is lack of scientific evidence that PRP injection is more effective or results in earlier return-tosport rates than other inert injections.

Although there are some reports about the use of PRP injection in the ACL reconstruction, they failed to provide any statistical difference in treatment outcomes between the treatment group and the control group. Sanchez et al. ${ }^{22)}$ have performed postoperative assessment in patients who had plasma rich in growth factors (PRGF) assisted $(\mathrm{n}=15)$ and the conventional ACL reconstruction $(\mathrm{n}=22)$ on arthroscopic and pathologic evaluation at 6 to 24 months. The use of PRGF influenced the histologic characteristics of tendon grafts, resulting in more remodeling compared with untreated grafts. However, arthroscopic evaluations were not statistically different between PRGF and control groups. Cervellin et al. ${ }^{23)}$ performed randomized control study about the application of autologous PRP gel to the donor site after ACL reconstruction with patellar tendon graft. At the 12-month follow-up, the postoperative evaluation using VAS and Victorian Institute Sport Assessment (VISA) scale and magnetic resonance imaging (MRI) evaluation was implemented on both autologous PRP treatment group $(\mathrm{n}=20)$ and the control group $(n=20)$. Although evaluation using VISA scale revealed that effectiveness of autologous PRP treatment was statistically significant, the VAS scale showed no statistical difference between the 2 groups. The recovery of the tibial and patellar bone defects by new bony tissues in both groups was equally successful and showed no statistical differences. The results of the study indicated no effectiveness of autologous PRP in subjective pain reduction at the donor site after the ACL reconstruction. Vogrin et al $^{24)}$ performed prospective randomized double-blind study on the effect of platelet gel on early graft revascularization after ACL reconstruction. After grouping those treated with platelet gel $(n=25)$ and those in the control group $(n=25)$, contrastenhance MRI was used to assess revascularization process in the osteoligamentous interface zone in the bone tunnel and in the intra-articular part of the graft. The treatment group showed faster revascularization in the osteoligamentous interface zone in the tibial bone tunnel, however, there was no statistical difference in the intra-articular part of the graft between the two groups. Nin et al. ${ }^{25)}$ performed prospective randomized study on 100 patients who had ACL reconstruction with allograft after dividing them into the PRP treatment group $(n=50)$ and the control group $(\mathrm{n}=50)$, and its evaluation was based on 2-year follow-up, radiologic, and MRI assessment. The 2-year follow-up assessment revealed that the use of platelet derived growth factor (PDGF) had no noticeable clinical or biomechanical effect. Orrego et al. ${ }^{26}$ studied the effects of platelet concentrate and a bone plug on the healing of hamstring tendons in a bone tunnel. After randomly dividing 108 patients requiring ACL reconstruction into control $(n=26)$, platelet concentrate $(n=26)$, bone plug $(n=28)$, and a combination of platelet concentrate and bone plug $(n=27)$ groups, MRI studies were prospectively carried out to compare the healing of hamstring tendons in a bone tunnel. The use of platelet concentrate only had enhancing effects on the maturation process of the graft but lacked effects on improvement in the osteoligamentous interface or tunnel widening evolution. Silva and Sampaio ${ }^{27)}$ also reported that treatment and control group showed no differences in signal intensity at the tendon bone interface on MRI at the 3-month follow-up. Despite some case or case series reports about the effect of PRP on accelerating the early recovery from tendon injury, PRP injection is currently not recommended in ACL reconstruction surgery, which is proven to have no efficacy in the human randomized controlled trials. 


\section{PRP for Articular Cartilage Defect or Osteoarthritis of Knee} Joint

Articular cartilage injuries and degenerative joint disease are one of the most challenging and actively researched field in orthopedic surgery and sports medicine. Woodell-May et al. ${ }^{28)}$ reported that the autologous protein solution (APS) prepared from PRP contained both anabolic (bFGF, TGF- $\beta 1$, TGF- $\beta 2$, EGF, IGF-1, PDGF-AB, PDGF-BB, and VEGF) and antiinflammatory (IL-1ra, sTNF-R1, sTNF-RII, IL-4, IL-10, IL13, and IFN $\gamma$ ) cytokines, and that the combination of these cytokines is a potential candidate for treatment of osteoarthritis. van Buul et al. ${ }^{29)}$ suggested that PRP influences conversion of human osteoarthritic chondrocytes by inhibiting the action of inflammatory cytokines such as IL-1 and NF-kB. However, despite the possibilities based upon the result of some in vitro studies, there is still a lack of evidence to support the effect of PRP in the treatment of osteoarthritis in humans.

In some studies, the combination of microfracture procedure and PRP injection in cases of osteochondral injuries showed that PRP injection was helpful in enhancing the effect of microfracture treatment, but the treatment effectiveness of PRP injection alone has not been studied yet. Milano et al. ${ }^{30)}$ performed experimental studies on the effect of autologous PRP with microfracture on chondral defects in a sheep model, and reported that treatment with PRP revealed advantageous effect on improvement of cartilage stiffness and showed higher International Cartilage Repair Society (ICRS) scores. Dhollander et al. ${ }^{31}$ has introduced a surgical technique about PRP gel application after microfracture procedure on 5 patients suffering from osteochondral patellar lesions in the knee and reported clinical improvement among the patients at the 24-month follow-up.

Intra-articular injection of autologous PRP has been increasingly implemented on patients with osteoarthritis and currently seems to be considered as one of the treatment options for osteoarthritis. Most of the studies on autologous PRP injection have been focused on the reduction of pain and improvement of function over time. Sampson et al. ${ }^{32)}$ reported the results of a pilot study about the PRP injection on 13 patients with primary or secondary knee arthritis, showing improvement in pain and symptoms without adverse effect on the scales of Knee Injury and Osteoarthritis Outcome Scores (KOOS). Wang-Saegusa et al. ${ }^{33)}$ reported improvement of VAS, SF-36, and Western Ontario and McMaster Universities (WOMAC) scores at the 6-month follow-up in 261 patients with OA symptoms more than 3 months who had 3 intra-articular injection of autologous PRGF at 2-week intervals. Filardo et al. ${ }^{34)}$ reported that 3 injections of intra-articular PRP in 90 patients with chronic knee degenerative conditions revealed improvement in Internatioal Knee Documentaion Comitee (IKDC) and EQ-VAS scores at the 2-year follow-up and that it had discernible positive effects especially on younger patients with early osteoarthritis. Napolitano et al. ${ }^{35)}$ also reported improved outcome by reviewing 27 patients with numerical rating scale (NRS) and WOMAC scores for 6 months. This study failed to show differences in degree of outcome compared with other studies because it simply evaluated the clinical results before and after the treatment without control. Sanchez et al. ${ }^{5}$ performed a retrospective cohort study on two groups who were treated with intra-articular injection of PRGF and with hyaluronic acid respectively and reported that PRGF treatment group showed improvement in pain and quality of life. Such effect was explained by action of PRGF in recovering the balance between intra-articular hyaluronic acid concentration and revascularization ${ }^{36}$. It suggested short-term results through a retrospective (evidence level IV) study with a small sample size. Kon et al. ${ }^{4)}$ reported that intra-articular PRP injection in 100 patients with chronic degenerative condition of the knee had positive effects on improving pain and quality life and on the scores of IKDC and EQ-VAS at the 1-year follow-up. Kon et al. ${ }^{37)}$ divided 150 patients with knee cartilage degenerative lesions or osteoarthritis into 3 groups, those treated with injections of autologous PRP $(n=50)$, low hyaluronic acid concentration $(\mathrm{n}=50)$, and high hyaluronic acid concentration $(n=50)$ and compared scores of IKDC and EQ-VAS among the groups at the 2-month and 6-month followup respectively. It was reported that the group with injection of autologous PRP had better outcomes in aspects of pain reduction and recovery of articular function. Because the study did not utilize randomized double-blind method, only suggested shortterm clinical reports and did not consider statistical bias, this study was inadequate to conduct statistical analysis due to lack of prominent differences in clinical results. Spakova et al. ${ }^{38)}$ reported a comparison study of PRP vs. hyaluronic acid in KellgrenLawrence grade 1,2, or 3 osteoarthritis patients with better result in PRP group. The authors concluded that their preliminary findings supported the application of autologous PRP as an effective and safe method in the treatment of the initial stages of knee osteoarthritis and further studies were necessary to confirm these results and to investigate the persistence of the beneficial effects observed.

Previous studies on intra-articular PRP injection reported its advantageous effects in pain reduction and function recovery, but their methodology is still questionable. There are no comparative 
results in the effectiveness of PRP injection to placebo or control injection in a double-blind method, nor with prolonged followup period. To date, there is still limited evidence about not only the effectiveness of PRP injection compared with other treatments on osteoarthritis but also strong recommendation toward PRP injection in treating osteoarthritis. Because of the cost-effectiveness, other treatments such as hyaluronic acid injection rather than PRP should be considered first. Studies need to implicate randomized double-blind using a proper control group to measure applicable treatment efficacy.

\section{Recommendation for Using PRP}

Since there is a lack of research evidence on treatment efficacy of PRP, preparation procedure, location and timing of injection, and amount of dosage to yield its clinical effectiveness has not been determined. The American Academy of Orthopedic Surgeons $(\mathrm{AAOS})^{39)}$ and WellCare Health Plans ${ }^{40)}$, Inc have announced the PRP protocols only for use in clinical trials and research, and that PRP is not recommended for treatment purposes by any conferences or institutions in the world.

\section{The Viewpoint of the Ministry of Health and Welfare} and Health Insurance Review and Assessment Service on Using PRP and the Legal Issue

It is required to know the review procedure of a new medical technology in Korea so as to discuss the legality of PRP. Currently, according to the systematic device rules (article 3 Section 6 of rules on New Health Technology Assessment), the validity and safety of a new medical technology (treatment and operation, medicine, medical materials, etc.) should be assessed prior to use by the Committee for New Health Technology Assessment (hereinafter referred to as nHTA) in the country. In contrast, in the past, while an assessment of a new medical technology was conducted, it could be used until the result of assessment was released. However, such use is prohibited by the medical law at present. Thus, it is required to grasp the assessment report of nHTA and the position of Health Insurance Review and Assessment Service (hereinafter referred to as HIRAS) so as to identify the legality of the currently operated PRP or a prolotherapy that uses it.

\section{Summary of the Report on the Assessment of Safety and Validity of PRP by nHTA}

1) Applied technology: PRP

PRP is an operation to promote the healing or regeneration of tissue by applying the concentrated blood plasma in which the platelet extracted from own blood to the part where bone is damaged or soft tissue should be regenerated. As per article 53 of medical law and article 3 of rules on New Health Technology Assessment, Autologous Platelet Rich Plasma Application, i.e., the new name of the terminology was applied on September 2, 2009.

2) Operation of nHTA and subcommittee

In the 10th nHTA on October 23, 2009, PRP was judged as a new health technology that should be assessed in consideration of the fact that the materials from own blood are actively studied in diverse clinical areas these days. The nHTA decided to assess PRP in a subcommittee by systematically studying the literature. Composed of 5 members, the subcommittee was held 4 times for 5 months from Dec 3, 2009 to Apr 5, 2010 to assess the same operation on the basis of the literature and submitted the result of study. The result of assessing safety and validity of PRP was finally deliberated in the 4th nHTA (2010.4.23) on the basis of the same contents.

\section{3) Assessment of PRP}

(1) Objective of assessment: The objective is to assess whether PRP is a safe operation and effective for healing or regenerating tissue.

(2) Assessment method: The literature search strategy to assess PRP selected the degree of healing or regenerating tissue or the term spent for this work as main medical results of PRP injection in patients with damaged bone or patients whose soft tissue should be regenerated. For the assessment of PRP, 8 domestic databases including KoreaMed and foreign databases like OvidMEDLINE, EMBASE and Cochrane Library were used. By searching strategy that uses the search word, 'platelet rich plasma', some 1,400 literatures were searched. Among the Korean and English literature in the proper type of study published after 2005 (systematic investigation of literature, random clinic test study), the animal test or preclinic test study and study which is not an original book and gray literature were excluded. Further, study on patients in the area of dental clinic (oral and maxillofacial surgery) and the study included in the systematic investigation of literature included in assessment were also excluded. The 1,391 units including redundantly searched literatures (396 units) were excluded and 9 studies (the systematic investigation of literature in 2 units, random clinic test study in 7 units) were finally included in the assessment.

(3) Safety: For safety, the death due to operation and complica- 
tion related to operation were assessed. The 2 systematic investigation of literatures reported that there was no problem in the safety of PRP. Although a clear causal relation between PRP and death was not identified, 1 case of death due to cardiac causes among those to whom PRP was administered was reported in a random clinic test study on cardiovascular disease patients. The incidence of complication in the group using PRP was higher than that in the re-combination human bone morphogenetic protein 7 administered group in a comparative study and the same in the cellulose/collagen operating group in another comparative study. Further, it was reported that the group using PRP showed a similar rate of complications with the group without the use of PRP. Although complications included hematoma, re-operation, edema, seroma, infection, etc., it was difficult to find the exact relation with PRP. Thus, the subcommittee concluded 1) it was difficult to find an exact relation between the occurrence of complication and PRP, because the study targets in the selected literatures were diverse and specific presentation was deficient, 2) it was difficult to make positive conclusion on the safety of PRP due to lack of literature that systematically verified safety.

(4) Validity: On the validity assessment, the degree of healing or regenerating tissue and its term were analyzed as main medical results.

The systematic investigation of 2 literatures reported that the effect of using PRP to heal fracture, chronic skin ulcer, surgical wound, etc does not have clinical ground. The group using PRP showed lower effect in bone adhesion in the comparative study of re-combination human bone morphogenetic protein 7 administered groups and there was a significant difference in healing ulcer on the chronic diabetes foot in the comparative study of cellulose/collagen operating group. Among 5 random clinic test studies that compared a group using PRP with another group without using PRP, 4 studies did not show significant differences in the degree of healing or regenerating tissue between the 2 studied groups. Although 1 literature showed results that PRP helped wound healing, 1) the study was a preliminary report, 2) the method of randomly allocating study targets was not specifically described, 3) due to the lack of comparison of equality between the test group and the contrast group at the commencement of study, it was difficult to generalize the results. Thus, the opinion of subcommittee was that it was difficult to verify the validity of PRP, because there was no significant difference in healing or regenerating tissue by using PRP in most studies.

(5) Suggestion: The nHTA commented that it was difficult to make a positive conclusion on the safety of "PRP" as per Article 3, Section 6 of new health technology assessment and the safety and validity of the technology was not supported yet, because the effect on healing or regenerating tissue was not verified. The result of deliberation by the nHTA and the result of check-up by the subcommittee were reported to the Minister of Health and Welfare on May 10, 2010.

\section{Position of HIRAS}

Based on the above assessment by the nHTA, the HIRAS stated that using PRP for a purpose other than study was illegal, because the safety and validity of treatments using PRP were not verified, adding that medical charges claimed to patients beyond the coverage of insurance may be reimbursed (currently examined by legal team of HIRAS). Asked whether Prolotherapy with PRP currently in use was possible as a kind of prolotherapy, the HIRAS also answered that it was illegal. However, the study on the PRP and similar operation is currently requested, it is still re-examined, leaving the possibility to recognize the validity of treatment in the other muscular and skeletal areas except for cartilage regeneration.

\section{Conclusions}

Scientific evidence has not been established to evaluate the efficacy of PRP treatment on knee joints, and the clinical use of PRP is not recommended worldwide. In Korea, PRP and Prolotherapy using PRP are defined as illegal medical technology whose safety and validity are not yet verified by the Ministry of Health and Welfare and HIRAS on the basis of the results of deliberation by the nHTA. Practicing physicians should remember that PRP injection to patients by imposing medical charge is still illegal as per the current medical law in Korea.

\section{References}

1. de Vos RJ, Weir A, van Schie HT, Bierma-Zeinstra SM, Verhaar JA, Weinans H, Tol JL. Platelet-rich plasma injection for chronic Achilles tendinopathy: a randomized controlled trial. JAMA. 2010;303:144-9.

2. Foster TE, Puskas BL, Mandelbaum BR, Gerhardt MB, Rodeo SA. Platelet-rich plasma: from basic science to clinical applications. Am J Sports Med. 2009;37:2259-72.

3. Mehta S, Watson JT. Platelet rich concentrate: basic science and current clinical applications. J Orthop Trauma. 2008;22:432-8. 
4. Kon E, Buda R, Filardo G, Di Martino A, Timoncini A, Cenacchi A, Fornasari PM, Giannini S, Marcacci M. Plateletrich plasma: intra-articular knee injections produced favorable results on degenerative cartilage lesions. Knee Surg Sports Traumatol Arthrosc. 2010;18:472-9.

5. Sanchez M, Anitua E, Azofra J, Aguirre JJ, Andia I. Intraarticular injection of an autologous preparation rich in growth factors for the treatment of knee OA: a retrospective cohort study. Clin Exp Rheumatol. 2008;26:910-3.

6. Felder-Puig R, Gyimesi M, Mittermayr T, Geiger-Gritsch S. Chemonucleolysis and intradiscal electrothermal therapy: what is the current evidence? Rofo. 2009;181:936-44.

7. Sisto DJ, Mitchell IL. UniSpacer arthroplasty of the knee. J Bone Joint Surg Am. 2005;87:1706-11.

8. Pak J. Regeneration of human bones in hip osteonecrosis and human cartilage in knee osteoarthritis with autologous adipose-tissue-derived stem cells: a case series. J Med Case Reports. 2011;5:296.

9. Engebretsen L, Steffen K, Alsousou J, Anitua E, Bachl N, Devilee R, Everts P, Hamilton B, Huard J, Jenoure P, Kelberine F, Kon E, Maffulli N, Matheson G, Mei-Dan O, Menetrey J, Philippon M, Randelli P, Schamasch P, Schwellnus M, Vernec A, Verrall G. IOC consensus paper on the use of platelet-rich plasma in sports medicine. $\mathrm{Br} \mathrm{J}$ Sports Med. 2010;44:1072-81.

10. Hall MP, Band PA, Meislin RJ, Jazrawi LM, Cardone DA. Platelet-rich plasma: current concepts and application in sports medicine. J Am Acad Orthop Surg. 2009;17:602-8.

11. Lopez-Vidriero E, Goulding KA, Simon DA, Sanchez M, Johnson DH. The use of platelet-rich plasma in arthroscopy and sports medicine: optimizing the healing environment. Arthroscopy. 2010;26:269-78.

12. Mehta V. Platelet-rich plasma: a review of the science and possible clinical applications. Orthopedics. 2010;33:111.

13. Paoloni J, De Vos RJ, Hamilton B, Murrell GA, Orchard J. Platelet-rich plasma treatment for ligament and tendon injuries. Clin J Sport Med. 2011;21:37-45.

14. Kajikawa Y, Morihara T, Sakamoto H, Matsuda K, Oshima Y, Yoshida A, Nagae M, Arai Y, Kawata M, Kubo T. Plateletrich plasma enhances the initial mobilization of circulationderived cells for tendon healing. J Cell Physiol. 2008;215:83745.

15. Anitua E, Sanchez M, Nurden AT, Zalduendo M, de la Fuente M, Azofra J, Andia I. Reciprocal actions of plateletsecreted TGF-betal on the production of VEGF and HGF by human tendon cells. Plast Reconstr Surg. 2007;119:950-9.
16. Murray MM, Palmer M, Abreu E, Spindler KP, Zurakowski D, Fleming BC. Platelet-rich plasma alone is not sufficient to enhance suture repair of the ACL in skeletally immature animals: an in vivo study. J Orthop Res. 2009;27:639-45.

17. Molloy T, Wang Y, Murrell G. The roles of growth factors in tendon and ligament healing. Sports Med. 2003;33:381-94.

18. Filardo G, Kon E, Della Villa S, Vincentelli F, Fornasari PM, Marcacci M. Use of platelet-rich plasma for the treatment of refractory jumper's knee. Int Orthop. 2010;34:909-15.

19. Coombes BK, Bisset L, Vicenzino B. Efficacy and safety of corticosteroid injections and other injections for management of tendinopathy: a systematic review of randomised controlled trials. Lancet. 2010;376:1751-67.

20. Hart L. Corticosteroid and other injections in the management of tendinopathies: a review. Clin J Sport Med. 2011; 21:540-1.

21. de Vos RJ, van Veldhoven PL, Moen MH, Weir A, Tol JL, Maffulli N. Autologous growth factor injections in chronic tendinopathy: a systematic review. Br Med Bull. 2010;95:6377.

22. Sanchez M, Anitua E, Azofra J, Prado R, Muruzabal F, Andia I. Ligamentization of tendon grafts treated with an endogenous preparation rich in growth factors: gross morphology and histology. Arthroscopy. 2010;26:470-80.

23. Cervellin M, de Girolamo L, Bait C, Denti M, Volpi P. Autologous platelet-rich plasma gel to reduce donor-site morbidity after patellar tendon graft harvesting for anterior cruciate ligament reconstruction: a randomized, controlled clinical study. Knee Surg Sports Traumatol Arthrosc. 2012;20:114-20.

24. Vogrin M, Rupreht M, Dinevski D, Haspl M, Kuhta M, Jevsek M, Knezevic M, Rozman P. Effects of a platelet gel on early graft revascularization after anterior cruciate ligament reconstruction: a prospective, randomized, double-blind, clinical trial. Eur Surg Res. 2010;45:77-85.

25. Nin JR, Gasque GM, Azcarate AV, Beola JD, Gonzalez $\mathrm{MH}$. Has platelet-rich plasma any role in anterior cruciate ligament allograft healing? Arthroscopy. 2009;25:1206-13.

26. Orrego M, Larrain C, Rosales J, Valenzuela L, Matas J, Durruty J, Sudy H, Mardones R. Effects of platelet concentrate and a bone plug on the healing of hamstring tendons in a bone tunnel. Arthroscopy. 2008;24:1373-80.

27. Silva A, Sampaio R. Anatomic ACL reconstruction: does the platelet-rich plasma accelerate tendon healing? Knee Surg Sports Traumatol Arthrosc. 2009;17:676-82.

28. Woodell-May J, Matuska A, Oyster M, Welch Z, O'Shaugh- 
nessey K, Hoeppner J. Autologous protein solution inhibits MMP-13 production by IL-1beta and TNFalpha-stimulated human articular chondrocytes. J Orthop Res. 2011;29:13206.

29. van Buul GM, Koevoet WL, Kops N, Bos PK, Verhaar JA, Weinans H, Bernsen MR, van Osch GJ. Platelet-rich plasma releasate inhibits inflammatory processes in osteoarthritic chondrocytes. Am J Sports Med. 2011;39:2362-70.

30. Milano G, Sanna Passino E, Deriu L, Careddu G, Manunta L, Manunta A, Saccomanno MF, Fabbriciani C. The effect of platelet rich plasma combined with microfractures on the treatment of chondral defects: an experimental study in a sheep model. Osteoarthritis Cartilage. 2010;18:971-80.

31. Dhollander AA, De Neve F, Almqvist KF, Verdonk R, Lambrecht S, Elewaut D, Verbruggen G, Verdonk PC. Autologous matrix-induced chondrogenesis combined with platelet-rich plasma gel: technical description and a five pilot patients report. Knee Surg Sports Traumatol Arthrosc. 2011;19:536-42.

32. Sampson S, Reed M, Silvers H, Meng M, Mandelbaum B. Injection of platelet-rich plasma in patients with primary and secondary knee osteoarthritis: a pilot study. Am J Phys Med Rehabil. 2010;89:961-9.

33. Wang-Saegusa A, Cugat R, Ares O, Seijas R, Cusco X, Garcia-Balletbo M. Infiltration of plasma rich in growth factors for osteoarthritis of the knee short-term effects on function and quality of life. Arch Orthop Trauma Surg. 2011;131:311-7.

34. Filardo G, Kon E, Buda R, Timoncini A, Di Martino A, Cenacchi A, Fornasari PM, Giannini S, Marcacci M. Plateletrich plasma intra-articular knee injections for the treatment of degenerative cartilage lesions and osteoarthritis. Knee
Surg Sports Traumatol Arthrosc. 2011;19:528-35.

35. Napolitano M, Matera S, Bossio M, Crescibene A, Costabile E, Almolla J, Almolla H, Togo F, Giannuzzi C, Guido G. Autologous platelet gel for tissue regeneration in degenerative disorders of the knee. Blood Transfus. 2012;10:72-7.

36. Anitua E, Sanchez M, Nurden AT, Zalduendo MM, de la Fuente M, Azofra J, Andia I. Platelet-released growth factors enhance the secretion of hyaluronic acid and induce hepatocyte growth factor production by synovial fibroblasts from arthritic patients. Rheumatology (Oxford). 2007;46:1769-72.

37. Kon E, Mandelbaum B, Buda R, Filardo G, Delcogliano M, Timoncini A, Fornasari PM, Giannini S, Marcacci M. Platelet-rich plasma intra-articular injection versus hyaluronic acid viscosupplementation as treatments for cartilage pathology: from early degeneration to osteoarthritis. Arthroscopy. 2011;27:1490-501.

38. Spakova T, Rosocha J, Lacko M, Harvanova D, Gharaibeh A. Treatment of knee joint osteoarthritis with autologous platelet-rich plasma in comparison with hyaluronic acid. Am J Phys Med Rehabil. 2012;91:411-7.

39. Santos F, Martinez. Practical guidelines for using PRP in the orthopaedic office [Internet]. Rosemont: American Academy of Orthopaedic Surgeons; c2012 [cited 2012 Apr 15]. Available from: http://www.aaos.org/news/aaosnow/ sep10/clinica3.asp.

40. WellCare. Clinical coverage guideline: autologous bloodderived growth factors [Internet]. Tampa: WellCare Health Plans Inc; c2012 [cited 2012 Apr 15]. Available from: http:// www.wellcare.com/WCAssets/corporate/assets/HS179_ Platelet_Rich_Plasma.pdf. 